\title{
Diversidad cultural, multilingüismo y reinvención de la educación intercultural bilingüe en América Latina
}

Luis Enrique López ${ }^{*}$

Mi opción por la lucha no tiene límites ni espacio: solo nosotros que llevamos nuestra causa en el corazón estamos dispuestos a correr todos los riesgos.

Rigoberta Menchú, 1985: 261.

\section{Resumen}

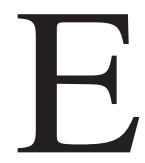

sta comunicación reevalúa algunos supuestos de la educación intercultural bilingüe en América Latina, sobre la base de una revisión breve de los contextos sociolingüísticos en los que este tipo de educación se desarrolla. Se privilegia los casos de Guatemala y Bolivia, países latinoamericanos en los que las poblaciones indígenas constituyen mayorías nacionales. Frente al desarrollo de la educación intercultural bilingüe, como parte de la oferta educativa estatal, se analiza el surgimiento de propuestas alternativas de líderes y organizaciones indígenas en busca de mayor pertinencia cultural. La divergencia surge,

${ }^{*}$ Docente del Programa de Formación en Educación Intercultural Bilingüe para los Países Andinos (PROEIBAndes), Universidad Mayor de San Simón, Cochabamba. 
en parte, de la poca importancia que hasta hoy los currículos escolares prestan a los conocimientos indígenas. Debido a ello, emergen propuestas de educación propia o endógena que, pese a su denominación autárquica, proponen también la interculturalidad y el plurilingüismo, relacionando así lo propio con lo ajeno. Pero, a diferencia de la educación intercultural bilingüe estatal, proponen que la educación en los territorios y comunidades indígenas esté bajo el control y la gestión indígena, aunque con financiamiento del Estado. Es gracias a propuestas como estas que, periódicamente, la educación intercultural bilingüe se renueva y reinventa en el continente en un movimiento que parece ir de abajo hacia arriba.

\section{Introducción}

Analizar la situación de la educación en las comunidades indígenas de América Latina nos remonta más allá de cuestiones exclusivamente pedagógicas, culturales y lingüísticas, pues en nuestra región las políticas nacionales que apoyan la aplicación de la educación bilingüe han sido en mucho el resultado del sufrimiento y la lucha indígenas en constante batalla contra el racismo y la discriminación. Paulo Freire (1973) estuvo en lo cierto cuando nos alertó, a fines de los sesenta y principios de los setenta, que la educación distaba de ser neutra y que cada modelo o estrategia educativa respondía a determinadas orientaciones políticas. En América Latina, la educación indígena ha estado siempre en entredicho, desde el momento en que nuestros países adoptaran los ideales liberales europeos, cuando nuestros libertadores, poco después de la independencia, suscribieron el postulado de la igualdad ante la ley.

Irónicamente, para los pueblos indígenas de América Latina el ideal de igualdad no significó otra cosa que la conculcación de algunas políticas proteccionistas instauradas 
en la colonia, con el consecuente abuso, pérdida de tierras, abandono y aun invisibilidad forzada que, de hecho, los colocó en condiciones aún peores en su relación con la tierra y el territorio que las que tenían durante el régimen precedente. Cabe recordar que, en general, los indígenas fueron excluidos del proceso independentista, en tanto la emancipación del yugo español fue dirigida principalmente por líderes políticos criollos o mestizos. Inspirados por la Revolución francesa, adoptaron los principios en los que se basaba el liberalismo europeo clásico que proyectaba la imagen de una entidad uniforme y homogénea. Por medio del castellano y de raíces socioculturales predominantemente hispánicas, buscaron construir una cultura nacional única, una sola nación y un país unitario y unificado. En América Latina, casi doscientos años después de la conclusión formal del régimen colonial, en unos países más que en otros, la relación entre indígenas y no indígenas se asemeja aun a la que existe en situaciones típicamente coloniales.

A la fecha, todas las sociedades amerindias continúan en la condición de comunidades subalternas (Spivak, 1988), aun en aquellos países en los que ellas constituyen la población mayoritaria, como en Bolivia y Guatemala. A pesar de que la legislación recientemente reconoció oficialmente la multiculturalidad y el multilingüismo y adoptó posturas y propuestas del multiculturalismo liberal, mucha gente, en especial los sectores sociales que detentan el poder, sigue percibiendo la diversidad cultural y lingüística como problema y como un obstáculo que compromete o impide la unidad nacional.

Pese a las condiciones desfavorables en las que viven, aún hay más de 40 millones de personas en América Latina (González 1994) que admiten su identidad indígena y/o hablan un idioma indígena. Si bien es difícil determinar con precisión quién es indígena y quién no lo es, así como 
lo que define esta categoría ${ }^{1}$, existe una alta correlación entre hablar un idioma indígena y ser indígena. De hecho, los latinoamericanos que no son indígenas pero que hablan una lengua indígena constituyen verdaderas excepciones que confirman la regla.

En Latinoamérica, los indígenas representan aproximadamente el $10 \%$ del total de habitantes de la región; pero, esta representación dista de ser uniforme y varía de país a país. En algunos, los indígenas ascienden a menos del 3\% e inclusive el $1 \%$ del total, como en los casos de Costa Rica y Brasil. Sin embargo, en otros países los indígenas constituyen verdaderas mayorías demográficas, tal es el caso de Bolivia y Guatemala, los dos países latinoamericanos con mayor presencia indígena.

\section{Guatemala y Bolivia}

Los últimos censos de población -2002 en el caso de Guatemala y 2001 en el de Bolivia- nos informan que en Guatemala más del $41 \%$ de la población nacional es indígena mientras que en Bolivia este porcentaje se eleva al $62 \%$ de los habitantes mayores de 14 años. A pesar de su incuestionable condición indígena mayoritaria, tanto en Bolivia como en Guatemala, hasta fines de la década de los 60 o principios de la de los 70 no se tomó previsión alguna respecto al uso escolar de la lengua materna del alumnado. Durante 300 años de régimen colonial y más de 180 de gobiernos republicanos, la población amerindia tuvo que aprender castellano casi a la fuerza. La clase dominante, por su parte, nunca hizo esfuerzo alguno por aprender idiomas indígenas, excepción hecha de los clérigos y los terra-

${ }^{1}$ La variable idioma ya no puede ser la única en definir la identidad indígena ya que en muchos casos, debido a la opresión colonial, los individuos y/o las comunidades que reclaman una identidad indígena bien pueden ya no hablar el idioma ancestral. 
tenientes que en las tierras altas necesitaban comunicarse con sus campesinos y tenían la motivación para aprender un idioma indígena y así ejercer control sobre sus nuevas tierras.

En esta comunicación tomo dos casos específicos -los de Guatemala y Bolivia- para examinar la implementación de la educación intercultural bilingüe (EIB) en la región. Para ello, aprovecho algunos hallazgos del observatorio sobre EIB en América Latina que llevamos a cabo desde el PROEIB Andes, en Cochabamba, Bolivia². En mi análisis, aprovecho también el trabajo de campo realizado recientemente en Guatemala y, por ende, me remito a entrevistas y a información recolectada durante charlas informales y en un taller realizado con profesionales y líderes indígenas (Marzo 2005).

Realzo que los líderes y las organizaciones indígenas cuestionen ahora algunas de las políticas nacionales que apoyan y promueven la implementación de la EIB, aun cuando se trate de políticas que ellos mismos contribuyeron a construir, a menudo desde la reivindicación y la protesta. Así, por ejemplo, en el caso boliviano, a principios de la década de los noventa el gobierno dictó nuevas disposiciones, recogiendo y aceptando demandas históricas de líderes y organizaciones indígenas respecto a la necesidad de cambios profundos en la educación. Como se verá, la mayoría de los argumentos que aquí presentamos provienen precisamente de lo que hoy en día declaran estos beneficiarios de la EIB.

\footnotetext{
${ }^{2}$ En el programa de maestría en educación intercultural bilingüe del PROEIB Andes se presta atención especial al desarrollo de la EIB en la región y se otorga particular énfasis a los cinco países que tienen la mayor presencia de pueblos indígenas en América Latina: Bolivia, Ecuador, Guatemala, México y Perú (cf. www.proeibandes.org).
} 
Guatemala cuenta con una historia relativamente larga de $\mathrm{EIB}^{3}$ que se remonta a fines de la década de los 60 , cuando se puso en práctica un proyecto educativo experimental con niños de las cuatro comunidades mayas más numerosas: kaqchikel, kiché, mam y qeqchí (cf. Dutcher 1995; Rubio, en prensa). La historia boliviana en este campo es más reciente y se remonta solo a fines de la década de los 70 y a principios de los 80 , cuando se implementaron dos proyectos piloto de educación bilingüe, uno en la región quechua y otro en el área aimara (cf. López, en prensa), siendo el quechua y el aimara los dos idiomas amerindios más hablados en el país. ${ }^{4}$

En Guatemala se hablan 24 idiomas diferentes, incluyendo el castellano; 21 pertenecen a la familia maya y los otros dos, garifuna y xinca, forman parte de otras familias lingüísticas. Mientras que, por lo general, los idiomas mayas gozan de una buena posición y se los usa activamente, pocos hablan el garifuna; aun cuando esta lengua cuente con muchos más hablantes en las costas caribeñas vecinas de Belice, Honduras y Nicaragua, o cuando otros más garífunas en la propia Guatemala se identifiquen como tales, sin hablar ya el idioma patrimonial de su comunidad. Por otro lado, no más de cuarenta xinca hablantes sobreviven en la actualidad y su idioma está al borde de la extinción. ${ }^{5}$

\footnotetext{
${ }^{3}$ Para facilidad de análisis y lectura me remito solo a la sigla EIB, en su condición de genérica y por ser hoy la más generalmente aceptada en todo el continente, pese a que precisamente en países como Guatemala se prefiera anteponer lo bilingüe a lo intercultural y se hable más bien de una educación bilingüe intercultural o EBI.

${ }^{4}$ No obstante, ya desde 1955 se llevaron a cabo proyectos de menor cobertura e incidencia nacional con distintas comunidades lingüísticas minoritarias, sobre todo de las tierras bajas situadas en la vertiente oriental de los Andes.

${ }^{5} \mathrm{El}$ xinca es un idioma no clasificado que solo se habla en Guatemala. Existe información contradictoria sobre la cantidad de xinca-hablantes. El Censo Nacional de Población de 2002 registra una población aproximada de 16000 individuos que se identifican como xinca pero los estudios especializados se refieren a no más de 40 hablantes de xinca.
} 
Cuando los proyectos de educación bilingüe comenzaron en Guatemala, el país atravesaba una crítica situación de violencia y guerra interna. En esa época, los niños indígenas solo llegaban a completar dos años de escolaridad en promedio y las niñas indígenas con dificultad terminaban el primer grado. El descuido en el cual el gobierno guatemalteco mantenía a niños y adolescentes indígenas era consecuencia de la persistencia de una sociedad racial y jerárquicamente estructurada. En dicha situación, como explícitamente lo declarara Rigoberta Menchú, uno "tenía una que ocultar su identidad [y su idioma] para poder resistir" (Menchú 1984:220). Pese a ello, hasta la fecha aproximadamente cinco de doce millones de guatemaltecos hablan un idioma maya, siendo la mayoría bilingües de maya y castellano, en muchos casos con el castellano como lengua de uso predominante o incluso ya como idioma materno. $Y$ es que el monolingüismo maya representa solamente el $13,5 \%$ de la población de más de 3 años de edad, restringiéndose principalmente a la población rural (Guatemala 2003) .

Guatemala tiene una de las tasas más altas de analfabetismo en Latinoamérica $-30 \%$ de los adultos no puede leer o escribir en escritura alfabética. La tasa de analfabetismo para los indígenas es del $47 \%$, el doble de la que muestra la población no indígena. Casi el $70 \%$ de las mujeres indígenas adultas que vive en áreas rurales no ha tenido la oportunidad de aprender a leer y escribir. De hecho, los guatemaltecos adultos aún tienen un bajo nivel general de educación formal, el promedio nacional apenas alcanza al quinto grado y los adultos indígenas solo completan un promedio de 2,6 años de escolaridad. Debido a las nuevas

\footnotetext{
${ }^{6}$ El monolingüismo en castellano representa el 65,5\% del total de la población guatemalteca, aproximadamente 10 millones de personas. El restante $21 \%$ se compone de bilingües o multilingües.
} 
políticas educativas implementadas durante la última década, la situación ha mejorado para todos los educandos menores de 18 años de edad, a pesar de que los estudiantes indígenas continúan notoriamente más rezagados que los monolingües en castellano, entre otras razones, precisamente debido a las limitaciones que experimenta la educación bilingüe oficial en Guatemala (Rubio, en prensa).

En Bolivia se hablan 33 idiomas indígenas diferentes pero no todos disfrutan del mismo estatus: millones hablan el quechua y el aimara (hay aproximadamente un millón y medio de aimaras hablantes y un poco más de dos de quechua hablantes ${ }^{7}$ ); algunos idiomas indígenas en las tierras bajas de la Amazonia y el Chaco cuentan únicamente con entre 100 y 70000 hablantes cada uno, pese a que algunos de ellos sean extremadamente vitales, como el araona en la cuenca amazónica; otros idiomas indígenas están prácticamente extintos, como el guasarug'we que solo tenía 30 hablantes en el año 2000 (cf. PROEIB Andes, 2001). Ante el riesgo de desaparición, algunos indígenas han optado por asimilarse, por la vía del matrimonio interétnico, a una comunidad vecina y lingüísticamente relacionada; este es el caso de los pakawaras que eran solo 21 en el 2000 (Ibid.).

En la actualidad, el $80 \%$ de la población boliviana habla castellano pero aproximadamente solo el $40 \%$ es monolingüe en este idioma; el $48 \%$ restante es bilingüe, trilingüe o multilingüe y habla uno o más idiomas indígenas. El castellano se ha extendido significativamente en los últimos 50 años. Antes de la revolución nacional de 1952, el mono-

\footnotetext{
${ }^{7}$ El aimara y el quechua también se hablan en otros países de Sudamérica: el aimara en tres (Bolivia, Perú y Chile) y el quechua en seis (Perú, Bolivia, Ecuador, Argentina, Colombia y Chile). La cantidad total de aimarahablantes es de aproximadamente dos millones y medio, y entre diez a doce millones de personas que aún hablan quechua. Hay aproximadamente dos millones y medio de quechuas en Bolivia y cerca a un millón y medio de aimaras viven en este país.
} 
lingüismo castellano representaba única y aproximadamente un $10 \%$ de la población. La revolución extendió el sistema escolar hispano hablante a las áreas más distantes del país. No obstante, el Censo Nacional de Población de 2001 reveló que por primera vez en la historia había cantidades significativas de pobladores urbanos que hablaban idiomas indígenas. Estas nuevas cifras, que nos refieren a más del $50 \%$ de la población en algunas ciudades bolivianas, muestran el bilingüismo y el multilingüismo que marcan a las ciudades bolivianas más importantes.

Aun cuando el analfabetismo es menor que en Guatemala, este es aún alto entre los indígenas bolivianos. La tasa nacional de analfabetismo es de $14,6 \%$, pero en áreas rurales -la mayoría de las cuales constituyen también reductos indígenas- esta se eleva a $26,2 \%$. El 37,9\% de las mujeres indígenas de más de 15 años de edad no ha tenido la oportunidad de aprender a leer y escribir; y, en este sentido, parece haber una situación paralela a la de las comunidades mayas de Guatemala. En el año 2000, los indígenas hombres en las áreas rurales completaron en promedio solo 6,9 años de escolaridad formal; tasa que se reduce a 5,7 años para el caso de las mujeres indígenas rurales (Nucinkis, en prensa). No obstante, se debe recordar que las mujeres indígenas bolivianas y también los hombres en algunas sociedades, son excelentes tejedoras y elaboran textiles complejos y detallados que, entre otras cosas, implican un elaborado conocimiento de aritmética y geometría y que en muchos casos representan también historias complejas y diferentes aspectos de la vida cotidiana (cf. López 2001).

Tanto en Bolivia como en Guatemala, en las últimas dos décadas, los pueblos indígenas se han tornado más visibles que nunca, habiendo su presencia política adquirido mayor relevancia en la vida política nacional. En Guatemala, este proceso comenzó a principios de los 90 y llegó a 
su climax con los Acuerdos de Paz de 1996, suscritos entre el gobierno y la guerrilla, después de 36 años de guerra interna. En Bolivia, el proceso comenzó antes, en 1982, cuando el país concluyó su larga historia de dictaduras militares, con el arribo al poder de un gobierno democrático que incluía a líderes de importantes organizaciones sociales, populares así como a representantes de ONG. Tanto en Guatemala como en Bolivia, profesionales y líderes indígenas populares socialmente reconocidos han alcanzado importantes posiciones gubernamentales. Tal es el caso, por ejemplo, de la Vicepresidencia de la República y de los ministerios de educación y de asuntos indígenas en Bolivia, y del Ministerio de Cultura y el Viceministerio de Educación en Guatemala. Casi un tercio de la Cámara de Diputados del Congreso Nacional boliviano está actualmente en manos de representantes indígenas. Se gesta una nueva constitución política del Estado para el año 2006 que podría concebir a Bolivia como un Estado plurinacional; los líderes y las organizaciones indígenas ya han comenzado su campaña a nivel nacional para garantizar su presencia en la Asamblea Constituyente Nacional que podría convocarse a principios de ese año y, al hacerlo, apelan a las redes sociales indígenas y no solamente al tipo de política imperante en Latinoamérica. Todo ello ocurre en un momento histórico en el cual el sistema político formal y el sistema de partidos que lo sustenta atraviesa su más severa crisis.

Los procesos que Bolivia y Guatemala experimentan forman parte de un fenómeno relativamente nuevo y multidimensional que, en mayor o menor grado, afecta a todos los países latinoamericanos desde el sur del Río Grande hasta la Patagonia. A fines de la década de los 80 y principios de los 90 la mayor parte de la región se involucró en reformas legislativas de alcance nacional, que conllevaron al reconocimiento de su origen indígena y a la aceptación de su actual condición multicultural y su composición multiétnica. 
Este reconocimiento ha implicado aun mayores revisiones conceptuales y pragmáticas de la naturaleza multilingüe y multicultural de todos estos países.

\section{Idioma y políticas en la educación bilingüe}

El castellano es el idioma oficial de Guatemala y la última Constitución de 1985 y la Ley de Educación de 1991 reconocen el derecho de los pueblos indígenas a la educación en su lengua materna (cf. www.mineduc.gob.gt). No obstante, el intento por reformar sustancialmente la constitución guatemalteca, a raíz de los Acuerdos de Paz de 1996, fracasó pues la mayoría de la población guatemalteca votó contra los cambios que incorporaban el reconocimiento de diversos derechos indígenas, incluyendo la oficialización territorial de todos los idiomas indígenas. Para contrarrestar en parte este fracaso nacional de reconocer su diversidad cultural y lingüística y los derechos indígenas, en el 2003 se aprobó una Ley de Idiomas Nacionales (cf. www.almg.org), se creó un Viceministerio de Educación Intercultural Bilingüe y a fines de ese mismo año un nuevo decreto gubernamental declaró la generalización de la EIB. Desde 1979, la educación bilingüe se ha extendido gradual y oficialmente bajo una estrategia de transición temprana. El proceso comenzó con solo 10 escuelas piloto. Hoy en día, la Dirección Nacional de Educación Bilingüe Intercultural (DIGEBI) tiene a su cargo 2193 unidades preescolares y 1200 escuelas primarias en las cuales se distribuyen materiales educativos en idiomas indígenas a alumnos de 13 diferentes comunidades etnolingüísticas mayas, aunque la atención sea mayor en los cuatro idiomas indígenas más hablados (kachiquel, kiche, mam y qeqchi). Existe también una Academia de las Lenguas Mayas de Guatemala (ALMG), de naturaleza autónoma pero de creación oficial, que se constituye en el único órgano nacional reconocido 
en cuestiones relativas a las lenguas y culturas mayas. La ALMG tiene filiales regionales en las 21 comunidades mayas, en las cuales promueve la difusión del alfabeto y la producción de materiales escritos en las lenguas indígenas respectivas (www.almg.org). No existe una estructura comparable para las otras dos comunidades étnicas (xinca y garifuna). Poco después de que se suscribieran los Acuerdos de $\mathrm{Paz}^{8}$, bajo presión diplomática internacional, el gobierno guatemalteco organizó una comisión paritaria de reforma educativa, con miembros del gobierno y de la sociedad civil, incluyendo profesionales y líderes indígenas reconocidos socialmente. Fue la primera vez que líderes e intelectuales indígenas se sentaban a una mesa de discusión oficial junto a sus ${ }^{9}$ contrapartes gubernamentales, para evaluar y revisar en profundidad las políticas y los programas educativos nacionales. La primera propuesta de reforma educativa se publicó en 1998 y desde entonces se han dado diversas iniciativas en las áreas de capacitación de maestros, desarrollo de material educativo e implementación de proyectos (cf. Comisión Paritaria, 1998). Las propuestas de la Reforma incluyen la educación intercultural para todos y la educación intercultural bilingüe. Sin embargo, las discusiones para configurar un nuevo programa educativo tomaron varios años pues fue difícil para las partes involucradas adaptarse a las nuevas variables de la diversidad lingüística y cultural. La nueva propuesta considera un diseño curricular en tres momentos y niveles diferentes: uno de alcance nacional, otro de cobertura regional -determinado en fun-

\footnotetext{
${ }^{8}$ Los Acuerdos de Paz de 1996 incluían un acuerdo específico titulado "Acuerdo sobre Identidad y Derechos de los Pueblos Indígenas" que enfatizaba la educación intercultural bilingüe, buscaba consolidar la educación maya, así como crear una Universidad maya. El Acuerdo de Identidad se firmó en 1995. ${ }^{9}$ En la sociedad guatemalteca, la población que tiene ancestro español o europeo o que no es indígena se considera ladina.
} 
ción al área sociolingüística correspondiente- y el último de carácter local, directamente relacionado con una comunidad y escuela específicas. Estos nuevos planes, empero, nunca llegaron a las escuelas; las ideas subyacentes a este diseño curricular y las de la estrategia simultánea de descentralización curricular parecen haberse abandonado, o por lo menos postergado. A principios de 2004, una nueva administración educativa decidió implementar, a partir de la gestión escolar 2005, solo el currículo escolar nacional y general, a través de la fórmula de un Currículo Nacional Base para el primer ciclo de la educación primaria -los dos primeros años de escolaridad primaria, construido con base en el conocimiento occidental y en la historia, cultura e instituciones oficiales de Guatemala ${ }^{10}$. Sin tomar en cuenta las declaraciones políticas generales y aún considerando las referencias al aprendizaje del primero, segundo y aun tercer idioma para todos, el nuevo programa guatemalteco es sin duda de carácter monocultural y parte del castellano cuando describe las competencias idiomáticas que los niños deben desarrollar en el primer idioma (Guatemala 2005).

Sin embargo, los resultados de las últimas pruebas nacionales pusieron en evidencia los efectos positivos de la educación bilingüe en el desempeño de los niños en la escuela primaria, cuando se compararon los resultados obtenidos por alumnos indígenas en escuelas bilingües con aquellos obtenidos por sus pares en escuelas exclusivamente hispano hablantes (Fernando Rubio, comunicación personal, marzo 2005). A raíz de ello, la misma ministra de educación se refiere ahora a la necesidad de revitalizar la educación bilingüe en el país y en abril 2005 lanzó una cam-

\footnotetext{
${ }^{10}$ En Latinoamérica en general, y hasta hace poco, los programas escolares oficiales solían considerar a las sociedades indígenas únicamente como parte del legado glorioso de la época prehispánica; pero en ellos, rara vez, se hacía referencia a la situación actual de estos pueblos.
} 
paña nacional para apoyar su fortalecimiento. Empero, el nuevo programa monocultural ya ha llegado a todas las aulas de las comunidades indígenas. Al respecto, la preocupación de las altas autoridades del sector se refiere únicamente al área de lenguaje y existe el riesgo que se abandone la perspectiva de interculturalidad adoptada por los Acuerdos de Paz (Entrevistas, marzo 2005). En el año 2004 esta nueva gestión gubernamental también promovió una campaña nacional para fortalecer una identidad nacional guatemalteca única, bajo lema "Orgullosos de ser guatemaltecos" (cf. www.mineduc.gob.gt), hecho que de por sí privilegia la guatemalidad y, al menos, disminuye, o de facto pone en cuestión, la posibilidad de identidades indígenas diferenciadas por pueblo.

En este contexto, hasta la fecha continúan las discusiones sobre el significado y la orientación del nuevo currículo pues para los especialistas en educación, y aun para los maestros indígenas, es difícil distanciarse de las antiguas prácticas de imposición de un programa nacional único desde el centro. Como ejemplo de esto, los primeros textos escolares para el nuevo programa de la escuela primaria se produjeron inicialmente en castellano y luego fueron traducidos, con ligeras adaptaciones, a los cuatro idiomas mayas más hablados, como resultado de la insistencia de mantener la política del texto oficial único para todos (Entrevista con varios educadores indígenas involucrados en el proceso, Marzo 2005). Aunque se aconsejó a los autores indígenas de dichos textos limitarse a traducir los materiales en castellano -política entendida localmente como de "contextualización", ellos tomaron, por su cuenta y riesgo, la decisión de modificar algunas secciones de estos materiales y adaptarlas culturalmente, para hacerlas más apropiadas y pertinentes (íbid.).

En Guatemala, el tema de la producción de textos en idiomas indígenas pone en evidencia una de las mayores dificultades con las que tropieza la EIB en América Latina en 116 
general, así como también ocurre en otras partes del mundo. Tal como en Guatemala, donde ha habido un cambio explícito en el discurso de la sociedad civil guatemalteca a favor de los enfoques interculturales en la educación (ibid.), en los últimos años se ha producido y divulgado, respecto de la educación inuit canadiense, una gran cantidad de fraseología sobre programas educativos culturalmente responsables y sensibles pero, en última instancia, los textos y exámenes para los estudiantes indígenas son los mismos que aquellos que rigen para cualquier otro estudiante del sur del Canadá (Milloy 1999). ${ }^{11}$

Oficialmente, la educación bilingüe guatemalteca también ha evolucionado retóricamente de una posición de transición hacia otra de mantenimiento y desarrollo, destacándose la necesidad de llevar la educación en lengua materna más allá de los primeros dos o tres grados de la primaria $^{12}$. No obstante, se debe recordar que el niño indígena promedio en Guatemala solamente completa dos a cuatro años de escolaridad y tiene también, en ese mismo período, una necesidad social acuciante de apropiarse del castellano.

Se ha incluido la EIB en algunos centros de formación docente para elevar la formación de los docentes sobre la naturaleza y las implicaciones de la vida y el trabajo en una sociedad multiétnica. 18 de aproximadamente 80 centros estatales de formación docente inicial ofrecen especialización en EIB, aunque todavía no se cubra todos los idiomas indígenas nacionales (Rubio, en prensa). ${ }^{13}$

\footnotetext{
${ }^{11}$ Agradezco a Tove Skutnabb-Kangas por dirigirme hacia esta referencia.

${ }^{12}$ En Guatemala se ofrece educación bilingüe solo en un grado de preescolar y en los primeros dos grados de la escuela primaria; en otras palabras, se la ofrece a niños entre los 5 y 7 años de edad o entre los 6 y los 8 .

${ }^{13}$ Además de estos 80 institutos públicos de formación de maestros, hay más de 250 instituciones privadas. La formación de maestros en Guatemala es todavía parte de la educación secundaria y comienza después de los primeros tres grados de la educación secundaria (el $9^{\circ}$ grado). Se está debatiendo incluirla en la educación superior, como en la mayoría de los países latinoamericanos.
} 
Cabe notar que en la última década el movimiento indígena maya ha cuestionado seriamente las estrategias oficiales de implementación de la educación bilingüe y en 1994 comenzó a implementar su propio modelo educativo maya en algunas escuelas. Este modelo maya incorpora estrategias anteriores y ya conocidas de la educación bilingüe pero, a diferencia de las perspectivas gubernamentales, destaca el conocimiento maya tradicional, por ejemplo, en el área de matemáticas. Uno de los aspectos más innovadores del movimiento educativo maya es la importancia que se le da a la filosofía maya, a su espiritualidad y forma de vida, así como a la participación comunitaria. Ellos consideran que la educación debe responder a su modelo alternativo de desarrollo que califican de: "económico, sociocultural y político [... y] profundamente enraizado en su identidad individual y colectiva" (CNEM 2004: 68). En este contexto, las escuelas mayas colocan un fuerte énfasis en la lengua y cultura mayas. Para 2005 había 56 escuelas mayas organizadas en dos redes escolares estrechamente relacionadas pero diferentes, ambas bajo el mandato político y educativo de un único Consejo Nacional de Educación Maya (Francisco Puac, comunicación personal, Marzo 2005).

Las diferencias entre el modelo oficial del gobierno y el del Consejo Maya son importantes. Por un lado, mientras la propuesta maya pone énfasis en el conocimiento local y el idioma indígena, la oficial presta mayor atención en la adquisición del castellano y en la así llamada cultura nacional guatemalteca, mediante un esquema de transición temprana. Por otro lado, mientras la estrategia oficial promueve una educación sólo en castellano para los grados $4^{\circ}$ y $5^{\circ}$, el modelo maya se postula para los diferentes niveles del sistema educativo, incluyendo ya un buen número de escuelas secunda- 
rias $^{14}$. De hecho, para ellos la educación bilingüe podría comenzar en cualquier nivel o grado y no necesariamente en el preescolar o el primero. Esta estrategia multinivel es el resultado del creciente interés de ancianos y padres de familia indígenas y aun de niños indígenas mayas en edad escolar que quieren ir a una escuela maya después de años de castellanización forzada (ibid.), posiblemente como una expresión de la militancia política maya. No obstante, debido a complejidades estructurales que la sociedad guatemalteca todavía no ha resuelto y a la secular historia de negar y descuidar su condición mayoritariamente indígena, ni la escuela oficial ni la maya pueden garantizar que todos los niños indígenas completen por lo menos la educación primaria (6 grados).

Las escuelas mayas podrían tener una ligera ventaja sobre la oficial debido a su naturaleza comunitaria y a la participación social en la gestión educativa, lo que en efecto podría resultar en más años de escolaridad, pero estas escuelas requieren negociar el reconocimiento oficial de sus estudiantes y establecer equivalencias con el sistema escolar gubernamental. Para avanzar en esta dirección, las escuelas mayas argumentan que se adhieren a los Acuerdos de Paz y a la Reforma Educativa que ellos mismos gestaron (cf. Comisión Paritaria 1998); quieren contribuir a este proceso con orientaciones, planes y estrategias de programa que posibiliten la operativización los ideales que planteó

${ }^{14}$ En una de las redes de educación maya bajo la supervisión del Consejo Nacional de Educación Maya -Asociación de Centros Educativos Mayas del Nivel Medio de Guatemala (ACEM) - 37 escuelas ofrecen servicios de educación secundaria a lo largo y ancho del país, siguiendo el modelo y las estrategias que se basan en ideales de multiculturalidad, interculturalidad y multilingüismo. ACEM fue fundada en 2003 bajo los principios de "una educación maya hacia la construcción de una sociedad justa, inclusiva e intercultural" (ACEM 2005:4). Algunas escuelas ACEM son privadas; otras están financiadas por comunidades mayas específicas y también reciben el apoyo de ONGs nacionales e internacionales. 
la Comisión de Reforma, y, así, retroalimentar el proceso de transformación desde la comunidad local y la práctica en aula (cf. ACEM 2005).

La convivencia de estos dos modelos podría contribuir a enriquecer las políticas nacionales de educación bilingüe puesto que, en el momento, la sociedad guatemalteca parece estar preocupada por mejorar la calidad de los planes educativos para los educandos indígenas. Parece que, por fin, los sectores no indígenas han comprendido que el desarrollo económico y social del país está en duda, precisamente debido a que la mayoría de los indígenas tienen acceso y permanencia limitados en el sistema educativo.

En Bolivia, la situación lingüística es en cierta medida diferente. La Constitución nacional no otorga estatus oficial a ninguno de los idiomas hablados en el país, aunque el castellano sea de facto el idioma oficial en todo el país. En el año 2001, como resultado de una nueva protesta indígena en las tierras bajas -el área de mayor diversidad étnica y lingüística, que abarca treinta y tres pueblos indígenas diferentes y treinta lenguas diferentes - se aprobó un decreto presidencial que reconoce oficialmente a los treinta y tres idiomas indígenas ${ }^{15}$ hablados en todo el país, incluyendo las otras tres lenguas andinas (López, en prensa). Pero, a este decreto no le siguieron políticas específicas para aplicar esta norma en el sector público y el acto de oficialización no implicó más que un cambio en la retórica del gobierno frente a la creciente presión social indígena. De hecho, la educación es hasta hoy el único dominio en el cual las políticas lingüísticas a favor de las lenguas indígenas están siendo implementadas por el gobierno boliviano. En forma creciente, sin embargo, desde la década de los 60, las culturas e

\footnotetext{
${ }^{15}$ Amén de los treinta idiomas que se hablan en las tierras bajas, otras tres lenguas -aimara, quechua y uru-se hablan en las tierras altas bolivianas, la región más densamente poblada.
} 
idiomas indígenas han disfrutado de mayor visibilidad y uso en la radio, televisión, videos y aun en el cine, como resultado de un creciente interés en el país por la problemática indígena. En los últimos cuatro años también se ha publicado y distribuido a nivel nacional un suplemento semanal de ocho páginas de un periódico de circulación nacional. Este suplemento incluye materiales sobre todo en las lenguas aimara y quechua, aunque también ha incluido textos en otros idiomas indígenas hablados en el país.

La Constitución reformada de 1994 define a Bolivia como un país multiétnico y multicultural aunque reafirma el carácter unitario de la nación (cf. www.minedu.gov.bo). Bajo ese marco, en 1994 se aprobó una nueva ley de educación que adoptaba la EIB como estrategia oficial a ser implementada con los pueblos indígenas, basada en el modelo de desarrollo y mantenimiento. La nueva ley también adoptó la política de la educación intercultural para todos, con la idea de que la organización jerárquica tradicional de la sociedad boliviana cambiaría sólo si el sistema educativo promovía un profundo cambio de actitud en todos los estudiantes y particularmente en los hispano hablantes. Esta nueva ley también abrió la posibilidad de que los educandos hispano hablantes aprendiesen un idioma indígena en la escuela, llevando así ciertos aspectos de la política de educación bilingüe al cauce de la sociedad hegemónica (cf. Hornberger y López 1997).

Como se mencionó, la ley de 1994 incluyó demandas que las organizaciones indígenas bolivianas y en general las sociales habían formulado desde 1982 y 1983 (cf. López, en prensa), al retorno a la democracia. A diferencia de Guatemala, el enfoque boliviano fue, hasta cierto punto, de abajo hacia arriba en el sentido que las demandas y la visión general fueron postuladas por representantes de los pueblos indígenas involucrados y por organizaciones de base. No fue el gobierno boliviano el que llevó la iniciativa 
en este campo, aunque igual que en Guatemala, el Ministerio de Educación había conducido proyectos piloto en educación bilingüe -a fines de la década de los 70 y principios de las de los 80 y 90-. De hecho, entre 1990 y 1995 se implementó el proyecto piloto boliviano más exitoso de educación bilingüe, cuando la Confederación Sindical Única de Trabajadores Campesinos de Bolivia (CSUTCB), otras organizaciones regionales indígenas ${ }^{16}$ y el sindicato de maestros rurales se unieron alrededor de la EIB. Estos sindicatos participaron en el diseño del proyecto, eligieron a los maestros y profesionales que luego nombraría el Ministerio de Educación y ejercieron control sobre las actividades del gobierno y de los maestros en las comunidades donde se realizaba el proyecto. Cuando se aprobó la nueva ley, los líderes indígenas consideraron que habían ganado una de las batallas más importantes hacia el reconocimiento nacional de uno de sus derechos fundamentales (íbid).

La nueva ley se puso en efecto en 1996, cuando la reforma educativa llegó a las primeras más de 1500 escuelas primarias a nivel nacional. Desde entonces, todas las escuelas rurales bolivianas de este nivel han sido incorporadas en la reforma; de estas, aproximadamente 2800 son bilingües $(22,2 \%$ del total) y cubren todos los ciclos de la educación primaria, que en Bolivia comprenden 8 años de escolaridad. No todas las escuelas rurales bolivianas, sin embargo, ofrecen los tres ciclos de educación primaria por lo que una gran cantidad de alumnos indígenas carece de la oportunidad de completar sus estudios básicos en la comunidad en la que viven. De hecho, aun un $19 \%$ de todas las escuelas rurales solo ofrecen los primeros tres grados;

\footnotetext{
${ }^{16}$ Dentro del marco general de este proyecto piloto quinquenal, la Asamblea del Pueblo Guaraní o APG jugó un papel determinante en la implementación de los planes de la EIB, en estrecha relación con su propio plan de desarrollo indígena, y ejerció su influencia a nivel nacional.
} 
no obstante, una mayor cantidad de niños rurales e indígenas pueden completar sus estudios de educación primaria yendo a comunidades y pueblos cercanos en donde se ofrecen los grados superiores. De cualquier modo, en los últimos años las tasas de conclusión de la primaria han ido creciendo significativa y aceleradamente entre la población indígena. En 2002, un $48 \%$ de los niños indígenas había completado sus primeros 8 años de escolaridad en comparación al $37 \%$ de 1997 . No obstante, las niñas indígenas rurales están aun en desventaja cuando se las compara con los niños pues solo el $43,2 \%$ de ellas completa la primaria (8 grados), mientras que la cifra de niños es del $53,3 \%$ (Nucinkis, en prensa).

En Bolivia se elaboraron materiales en tres de los idiomas indígenas más hablados en Bolivia, quechua, aimara y guaraní ${ }^{17}$ para los primeros seis grados de la escuela y existen ya planes para introducir este nuevo enfoque educativo entre grupos más pequeños en las tierras bajas. Es posible que la educación bilingüe se implemente en el nivel secundario en el año 2006.

Casi la mitad de los institutos de formación docente (10 de 22) se han transformado de centros de formación exclusivos para una educación monolingüe en castellano en centros bilingües. También se ha implementado una nueva política nacional de participación popular en educación que, entre otras medidas, implica la organización de cuatro Consejos Nacionales de Educación de los Pueblos Indígenas, responsables de ejercer control social y escrutinio sobre la

\footnotetext{
${ }^{17}$ Aunque más gente habla el chiquitano (aproximadamente 120.000) que el guaraní (ca. 80 000), la educación bilingüe oficial comenzó en territorio guaraní debido a la presión política y al nivel de organización social y étnica de los guaraníes (cf. López, Luis Enrique. 1997. "To Guaranize: A Verb Actively Conjugated by the Bolivian Guaranis”. En N. Horberger (ed.) Indigenous Literacies in the Americas, International Journal of the Sociology of Language. Berlin/New York: Mouton de Gruyter. 321-53.).
} 
implementación y práctica de las políticas educativas y lingüísticas nacionales. Estos consejos indígenas son también producto de las demandas y reclamos indígenas.

Diez años después de que estas nuevas políticas educativas y lingüísticas fueran puestas en práctica, los líderes y las organizaciones indígenas, entre ellos los Consejos Educativos de Pueblos Originarios que acabamos de mencionar, han evaluado la implementación de la EIB en el país para establecer los logros y limitaciones de su transformación a política oficial y nacional (cf. CEPO 2004). En general, consideraron que habían cometido un error al pensar que la situación sociopolítica se transformaría radicalmente con la dación de nuevas leyes y reglamentaciones gubernamentales. El liderazgo indígena está ahora convencido de que aunque la legislación ayuda, esta no basta; pues se necesitan profundos cambios de actitud para superar el racismo y la exclusión social y económica a la que los pueblos indígenas se encuentran sometidos, producto de la condición colonial persistente en el país (íbid.). Hoy, también los líderes de algunas comunidades indígenas cuestionan parcialmente la aplicación del modelo oficial de la EIB que sus líderes y dirigentes ayudaron a diseñar, a través de sus reivindicaciones.

Aunque no hay todavía un movimiento social indígena similar al que dio origen a las escuelas mayas en Guatemala, varios líderes indígenas bolivianos se refieren ahora a una estrategia educativa que sea no solo intercultural y bilingüe sino también productiva y que se inserte más profundamente en las formas indígenas de pensar, ser, sentir y actuar (íbid.). Estas nuevas demandas surgen de comunidades mayoritarias, como la quechua y la aimara, así como también de comunidades lingüísticas con menor población como las orientales y amazónicas. Una inquietud común es que después de diez años de aplicación de la reforma educativa y de más de quince de introducción de la EIB desde 
el Estado, el Ministerio de Educación no ha logrado aún potenciar el desarrollo de programas curriculares locales y descentralizados, y ha abocado todos sus esfuerzos a diseñar e implementar el tronco común curricular de alcance nacional (ibid.) ${ }^{18}$. Ello los ha puesto suspicaces y los ha llevado a concluir que al gobierno nacional solo le interesa recurrir a una forma única de educación y a un fondo igualmente único de conocimiento (Moll y Greenberg, 1990): el occidental y hegemónico. Ello ocurre en un contexto en el cual, el propio tronco común busca ser intercultural y propicia la recuperación de algunos saberes y prácticas locales, lo que parece estar ahora en cuestión es la discusión no sobre aspectos y elementos aislados sino sobre la matriz cultural de la cual parte la propuesta curricular oficial.

Las nuevas demandas indígenas bolivianas se asemejan al espíritu del movimiento de educación maya o al de las organizaciones indígenas colombianas que demandan una educación enclavada en su propio proyecto de desarrollo o "plan de vida", como allí se le denomina. Esto puede haber motivado el título de su nueva propuesta educativa: "En línea con la educación indígena; hacia la autodeterminación ideológica, política, territorial y sociocultural" (cf. CEPO 2004). En este nuevo contexto, es común ahora que los líderes de las diferentes comunidades indígenas latinoamericanas se refieran a la importancia de su educación propia -un modelo endógeno de educación que, contrariamente a lo que uno podría esperar, no responde a una motivación aislacionista o separatista. Precisamente, debido al contex-

\footnotetext{
${ }^{18}$ Las reglamentaciones del programa boliviano prescriben la existencia de un programa curricular nacional central y común -el llamado tronco común-- y de una serie de ramas diversificadas de gestión local. Este programa central considera las competencias que todo alumno boliviano debe desarrollar en la escuela y el programa diversificado se refiere a las competencias locales y al contenido local y está, por ende, más estrechamente relacionado con los fondos de conocimiento indígena.
} 
to de contacto y conflicto cultural y lingüístico en el cual históricamente se ha venido construyendo y reproduciendo el conocimiento local es que estos líderes indígenas quieren que su educación sea intercultural y bilingüe y, por ende, flexible y abierta frente a otros idiomas y fondos o tradiciones de conocimiento. Pero, lo que más está en cuestionamiento es la problemática del control social de la educación y del manejo de la escuela. Ellos quieren ahora ver la escuela como realmente suya, como parte integral de la comunidad indígena y no como una institución ajena y aislada como lo ha sido desde su inscripción física en la comunidad desde fines del siglo XIX y a lo largo del XX. Más aún, quieren intervenir en la toma de decisiones sobre su funcionamiento y el tipo de educación que imparte. Se trataría no de una escuela para su inserción o asimilación a la sociedad hegemónica sino más bien de una institución que potencie un sentimiento de ciudadanía diferenciada o étnica, sentimiento complementario al de la ciudadanía nacional.

Los yuracarés nos proporcionan un ejemplo de esto, en su condición de hablantes de un idioma no-clasificado y de una comunidad etno-lingüística que solo entró en contacto con la sociedad hegemónica hace cerca de 50 años cuando una misión protestante buscó asimilarlos al cauce de la corriente dominante boliviana que solamente habla castellano. Únicamente 3500 yuracarés sobreviven hoy en aldeas y caseríos indígenas en los bosques tropicales sudamazónicos, aunque muchos de ellos viven también en pueblos y ciudades intermedias de la región amazónica. Parte de la comunidad yuracaré, que parecía lingüística y culturalmente asimilada, ha decidido volver a "la etapa anterior"; ahora quiere que sus hijos recuperen el idioma y la cultura ancestral y trata de restaurar su uso en la vida cotidiana de la comunidad local, como parte de un proyecto mayor de reafirmación identitaria yuracaré. Quinientos yuracarés han decidido volver a asentamientos en la selva, deman- 
dando derechos territoriales en el marco de actual legislación boliviana que reconoce el derecho indígena a sus tierras comunitarias de origen (TCO). Como parte del reclamo y reafirmación de su derecho a una TCO han decidido establecer sus propias escuelas yuracarés. En el año 2004 pidieron al PROEIB Andes que los ayudaran a sistematizar sus propuestas educativas y capacitar a sus maestros para poder iniciar su "propio" proyecto de educación; un proyecto que si bien manejado por su comunidad, debería recibir fondos fiscales para su funcionamiento. En ese marco, no reivindican más la EIB oficial, tal cual es implementada por el Ministerio de Educación; en su lugar han decidido organizar su propia educación yuracaré, la misma que consistiría de lo que se podría llamar un sistema educativo dual -en las mañanas replicarían la mejor educación oficial posible, con textos y materiales de la reforma educativa en castellano, maestros titulados e inclusive supervisión y apoyo oficial; mientras que en las tardes construirían un sistema educativo paralelo y alternativo dirigido a la recuperación lingüística y cultural bajo la dirección de maestros de la comunidad elegidos por ellos y bajo control comunitario y organizativo yuracaré.

La propuesta yuracaré se asemeja de alguna manera al modelo de doble vía del así llamado modelo aborigen australiano (cf. Harris 1990), pero difiere de este en cuanto a que los yuracarés consideran que este esquema es mejor que el modelo oficial de la EIB, pues opinan que no se puede alcanzar o tan siquiera buscar llegar a la interculturalidad si antes no se fortalecen o reposicionan la cultura y el conocimiento indígenas locales, tanto a nivel nacional como ante los propios ojos de sus miembros. Dicha relocalización de los idiomas y las culturas indígenas resulta contrahegemónica frente a la mentalidad colonial persistente en el país, e inclusive implica la superación de situaciones en las cuales miembros de los mismos pueblos indígenas han adoptado ya ciertas perspectivas coloniales. 
Desde situaciones similares, es ahora común que otros líderes indígenas bolivianos mencionen la necesidad de orientar la educación intraculturalmente antes de hacerlo interculturalmente; es decir, comenzar por la recuperación y sistematización del conocimiento ancestral (Froilán Condori, comunicación personal, noviembre 2004). Así, desde la intraculturalidad se estimularía la confianza y la creencia en el legado cultural propio y también se contribuiría a que las sociedades indígenas subalternas en cuestión desarrollen su autoestima y una imagen positiva de sí mismas. Como postulan algunos profesionales indígenas en Guatemala, dicha intraculturalidad podría, incluso y hasta cierto punto, implicar un uso estratégico, político $y$, por ende, consciente de algunos tipos de esencialismo indígena (Rodrigo Chub, comunicación personal, marzo 2005).

\section{Una nueva visión de la educación intercultural bilin- güe}

Para entender la razón por la cual algunos líderes indígenas bolivianos cuestionan ahora el modelo educativo que antes reclamaban y que, de hecho, ayudaron a diseñar o el motivo por el cual los líderes mayas guatemaltecos expresan diplomáticamente sus reservas sobre las estrategias oficiales de implementación de la educación bilingüe, es necesario referirse a la distinción entre las dimensiones política y pedagógica de la EIB que los ministerios de educación usualmente establecen. Los ministerios generalmente conciben la EIB como una modalidad educativa que contribuye al mejor desempeño del estudiante y a mejores las condiciones pedagógicas en general, mientras que los líderes y las organizaciones indígenas la perciben más bien como una estrategia integral bien establecida en su lucha histórica por la supervivencia como sociedades diferentes y por terminar con su exclusión política y económica. Desde 
esa perspectiva, la EIB es vista como herramienta que facilita el acceso al poder. Mientras que para los que toman decisiones a nivel gubernamental, la EIB influye positivamente en los resultados del aprendizaje, para las organizaciones y líderes indígenas la contribución más importante de la EIB es el desarrollo de la autoestima y la construcción de identidades indígenas diferenciadas en el marco de la lucha contra la opresión colonial y el racismo, pero también por la apropiación de conocimientos.

La EIB es el camino hacia nuestra propia educación, que a la larga nos permitirá situar nuestra lucha en la disputa por el poder formal. Como instrumento de nuestra liberación, la EIB nos permitirá construir una sociedad justa y equitativa donde se respeten esos derechos con los que ahora solo podemos soñar. No queremos un conflicto armado, postulamos una lucha hacia la apropiación de conocimientos. (Walter Gutiérrez, Presidente del Consejo Educativo Aimara, de Bolivia, entrevista, Octubre 2004).

Las entrevistas a líderes indígenas destacan que el cambio educativo no puede verse aisladamente; la educación es un derecho y como tal está relacionada a con otras prerrogativas que son igualmente importantes para el bienestar indígena, como el derecho a la tierra y al territorio, el derecho a los recursos naturales y el derecho al propio idioma, al conocimiento propio, a la propia historia y a las propias formas de ser, sentir y actuar. Resulta igualmente imperativo para ellos aprender un segundo idioma y ganar acceso a otros fondos de conocimiento necesarios para interactuar con miembros de otras sociedades (cf. CEPO 2004).

En forma más general, parece ser que, cuando la EIB se convierte en política nacional, pierde o al menos se oscurece parcialmente el significado y contenido polí- 
tico subyacente que los líderes indígenas le reconocen. Empero, esto no significa que en el pasaje de una demanda comunitaria u organizacional indígena a una política gubernamental todo se haya perdido. En América Latina, el reconocimiento gubernamental y oficial es ciertamente una ventaja que todo líder indígena quiere lograr y de hecho demanda, pero los líderes indígenas resienten que la EIB se entienda e implemente como una medida aislada relacionada sólo con la esfera educativa. Les resulta aún más preocupante que la EIB se vea como un modelo únicamente para indígenas. Los líderes guatemaltecos y bolivianos aseveran de modo explícito que este no debería ser el caso:

No estamos hablando de un modelo educativo solo para mayas. La educación bilingüe necesita ser extendida también a todas las escuelas ladinas y beneficiar a los niños ladinos también. Siempre hemos sido bilingües y multilingües y sabemos que hay ventajas en hablar más de un idioma. Depende de ustedes ahora aprender nuestros idiomas y acrecentar su capital lingüístico. (Francisco Puac, comunicación personal, marzo 2005)

La EIB necesita transformarse en un modelo de dos vías: de un idioma indígena al castellano y del castellano a un idioma indígena. Todos en Bolivia necesitan aprender nuestros idiomas en tanto nosotros hemos tenido que aprender y usar el castellano. De una vez por todas necesitamos erradicar el monolinguismo y el monoculturalismo si queremos que las condiciones políticas y sociales de este país mejoren y si queremos que nuestra democracia incluya a todos y sea intercultural. (Walter Gutiérrez, comunicación personal, septiembre 2004)

No obstante, tanto en Bolivia como en Guatemala, los líderes y las organizaciones indígenas aún apoyan y defienden el modelo oficial de la EIB pues lo consideran superior al modelo monolingüe y monocultural precedente (CEPO 2004; Francisco Puac, comunicación personal). 
Empero, ahora consideran que el tipo de EIB que se está implementando no es más que la oferta educativa estatal para los educandos indígenas; con ello consideran que se corre el riesgo de que esta se convierta también en una modalidad compensatoria, de segunda clase y, en suma, de una educación subalterna supeditada siempre al modelo educativo hegemónico.

En Bolivia, diez años después de que las organizaciones y líderes indígenas lograran inscribir la EIB en la legislación y las políticas linguoeducativas nacionales, los líderes y las organizaciones indígenas ven necesario avanzar hacia una profundización de la EIB, que incluye su extensión hacia las poblaciones hispano-parlantes, ejerciendo el poder ganado hasta el momento a raíz de su propia participación política y de su participación parcial en la toma de decisiones en el campo de la EIB. Los líderes indígenas son conscientes del poder que sus demandas tienen ante el gobierno.

La minoría blanca y mestiza en el gobierno también sabe que implementar la EIB y ceder, en parte, a las presiones indígenas les permite mantener su parte del pastel; en ese marco este tipo de educación se convierte en concesión o reacción parcial a la compleja situación política y social aun no resuelta que la minoría en el poder heredó y que está aún lejos de resolver. Ambas partes saben lo que pueden esperar, lo que necesitan negociar y hasta dónde pueden llegar, en la medida que tratan de reinterpretar las condiciones multiétnica, plurilingües y multiculturales de su sociedad y en el toma y daca de la construcción de relaciones nuevas y más democráticas entre los sectores indígenas y no indígenas de la sociedad.

El hecho es que a líderes y organizaciones indígenas les preocupa no únicamente la educación de sus niños indígenas sino también la de todos los niños bolivia- 
nos y guatemaltecos; por ello es que de forma periódica expresan su inquietud sobre la educación que se ofrece a todos los miembros de los países de los que ahora son parte. Según ellos, la EIB debería abarcar también a la población blanca y mestiza y desde una educación crítica todos deberían tomar conciencia de la problemática indígena, incluyendo su identidad, valores y aspiraciones etnolingüísticas.

Para que los idiomas indígenas se valoren, a los estudiantes hispanohablantes debería enseñárseles al menos lo básico del idioma indígena más hablado en la región en la que viven. Los líderes indígenas consideran que si las relaciones y estructuras de poder en estas sociedades multilingües han de transformarse, la población hegemónica también debe ser bilingüe y abandonar su histórico monolingüismo y monoculturalismo en castellano. Asimismo, están muy conscientes de que la EIB solo mejorará y se sostendrá cuando también se la ofrezca a quienes hoy disfrutan y ejercen el poder. De otra forma, la EIB seguirá siendo un modelo educativo de segunda clase o aún peor, un tipo de educación que se ofrece únicamente a los pobres. De allí que, desde su perspectiva, no es solo una cuestión de equidad sino también y principalmente un asunto de igualdad, puesto que la EIB también es vista como vía a una ciudadanía distinta y renovada.

En otras palabras, la educación lingüística en las sociedades multilingües de América Latina tiene que abandonar la visión compensatoria en la cual se situó en sus inicios. En tiempos de democracia y de la emergencia de voces indígenas, cada vez más altisonantes, la EIB debe ser vista como un modelo educativo capaz de contribuir al enriquecimiento cultural de cada miembro de la sociedad. Si a finales de la década de los 80 la educación bilingüe latinoamericana giró de una estrategia transitoria, hacia una salida un tanto improvisada de buscar desarrollar y mantener los idiomas indígenas, en este nuevo siglo podría ser necesario 
poner en vigencia una educación bilingüe de enriquecimiento y de doble vía: una educación en la cual se dé tanta importancia al idioma indígena, así como al conocimiento indígena y a la visión del mundo que lo subyacen, como obviamente también al castellano o al portugués, y al conocimiento occidental, en un contexto en el cual todo estudiante se beneficia de la EIB. Sin embargo, la instauración de una educación intercultural bilingüe para todos tampoco excluye que los líderes y las organizaciones indígenas busquen luego modelos y estrategias educativas alternativas que, pese a ser interculturales y bilingües, puedan muy bien apuntar en forma gradual a una relevancia cultural cada vez mayor en su lucha por acrecentar su reconocimiento político y su emancipación social. El recorrido de la EIB pone en evidencia la estrategia indígena de avanzar por aproximaciones sucesivas hacia modelos educativos cada vez más críticos y radicales.

La historia de la EIB a la fecha nos lleva a considerar que las futuras demandas indígenas pondrán énfasis en la filosofía y el conocimiento indígenas puesto que lo que recientemente ha estado en juego, tanto en Guatemala como en Bolivia, ha sido la dignidad como seres humanos $y$, en relación con ello, la controversia entre fondos diferentes y conflictivos de conocimiento. Sin embargo, como se ha recalcado repetidamente, "No podemos pensar en un modelo de aislamiento. No caeremos en la misma trampa de exclusión que la sociedad hegemónica ejerció sobre nosotros por siglos. Es muy diferente pensar en la necesidad de recuperar y reforzar lo que es nuestro, porque para nosotros ese es solo el primer paso hacia la interculturalidad. Estamos conscientes de que ahora también necesitamos estar en posesión del conocimiento ladino-occidental pero queremos ejercer nuestro derecho a escoger y necesitamos tener la opción de elegir críticamente lo que se nos adapta mejor. Estamos en contra de un modelo de desarro- 
Ilo que malinterpreta nuestros pensamientos y conocimientos, un modelo que sólo persigue la acumulación económica y nuestra asimilación en la forma de vida occidental hegemónica" (Francisco Puac, comunicación personal, marzo 2005).

Cuando en Bolivia y Guatemala, la EIB trascienda las comunidades indígenas donde ahora se implementa, se tendrá que pensar también en otros modelos y estrategias que respondan a escenarios sociolingüísticos complejos y diversos, tanto en comunidades rurales como en pueblos y ciudades. Si la EIB llega a las escuelas, aun si es débilmente, los educandos podrán ver el contenido alternativo que portan los conocimientos, valores y prácticas sociales indígenas. Entonces, Latinoamérica se enfrentará con la necesidad de volver a escribir su historia y simultáneamente concebir la diversidad como un valor en sí mismo.

\section{5. ¿Qué implica esto para la reinvención de la EIB en nuestra multilingüe América?}

En primer lugar, significa colocar la problemática del lenguaje en una dinámica ecológica, donde los idiomas indígenas no sean vistos en aislamiento ni desligados de la historia que les ha tocado recorrer, desde que les cupo compartir espacios con el castellano. Tampoco puede verse a las lenguas desvinculadas de las inquietudes y expectativas de sus hablantes respecto a su lugar en la vida y a su futuro como miembros de comunidades etnolingüísticas específicas y a la vez diferentes de otras. Es preciso prestar atención a la historia lingüística de cada sociedad multilingüe, pues docentes y educandos tienen que comprometerse en el proceso de revisión crítica de la situación de contacto y conflicto idiomáticos que caracteriza a cada escenario multilingüe. Analizar los factores que determinan, por ejemplo, la desventajosa situación de un idioma indígena determina- 
do, frente al idioma europeo dominante, con el cual converge, empoderará a los educandos indígenas, y puede incluso contribuir a que los estudiantes de los sectores hegemónicos tomen conciencia de los factores históricos que determinan la opresión de un idioma y, con ello, su decadencia y pérdida. Por lo tanto, una comprensión lingüística que analiza y describe al lenguaje sin considerar su contexto social y las complejidades sociolingüísticas y políticas del multilingüismo resulta ser totalmente inapropiada.

Ciertamente, se requiere situar a los idiomas indígenas en un contexto multilingüe de aprendizaje y uso idiomático. Esto nos permitirá, por un lado, reinscribir los idiomas indígenas en la larga historia de convergencia y contacto de los múltiples idiomas que caracterizan a esta parte del mundo. Por otro lado y en conexión con este primer argumento, es necesario dejar de entender el bilingüismo, o el multilingüismo si es que vamos hacia eso, como la simple suma de dos o más monolingüismos. Si esto ya dejó de ser cierto en los círculos académicos latinoamericanos, entre quienes ponen en práctica la educación intercultural bilingüe y entre los maestros que la aplican prevalece el mito de que los idiomas deben mantenerse lo más separados posibles el uno del otro, aún en casos en que los niños están expuestos a dos o más idiomas desde su nacimiento y recurren simultáneamente a dos o más idiomas para comunicarse con sus pares en la vida cotidiana. Si bien es cierto que muchas veces dicho entendimiento surge de la necesidad de revitalizar el idioma más débil de una comunidad, así como de contrarrestar la hegemonía lingüística del castellano, se necesita prestar cuidadosa atención a la naturaleza multilingüe de la comunidad misma y al carácter particular de la comunicación en una comunidad bilingüe y entre individuos que también lo son. En otras palabras, un enfoque ecológico para la educación lingüística tendría que examinar y al mismo tiempo responder a la manera en que se usa 
un idioma dado en conexión con otros idiomas en un escenario social multilingüe.

Más aún, los idiomas indígenas no deberían tratarse en aislamiento o separados de los territorios en los cuales se los habla y usa. Es esta perspectiva territorial y localizada del lenguaje la que nos permite restaurar y comprender la cercana relación existente entre lengua y cultura; y vincular estrechamente los idiomas con los valores, conocimientos, prácticas culturales y aún sentimientos de sus hablantes.

Los pueblos indígenas se aproximan a la vida desde una perspectiva global y holística y tienden a ver la complementariedad de diferentes aspectos de la vida. Las comunidades multilingües y las escuelas bilingües deben aproximarse a la enseñanza del lenguaje desde esta perspectiva integral. De allí que también se requiera un giro epistemológico en la EIB, pues es necesario distanciarse de la tradición positivista que tiende a dominar la enseñanza del lenguaje para acercarse a un enfoque interdisciplinario y más intuitivo y émico, que nos acerque a la manera en la que la propia familia indígena y sus miembros concibe el aprendizaje y la enseñanza idiomática; desde esa comprensión será posible negociar modos y formas de enseñanza que a la postre resulten social y culturalmente más apropiadas en los contextos indígenas. Asimismo, el aprendizaje del lenguaje deberá superar su orientación exclusivamente lingüística y pedagógica y restablecer y revisar las relaciones entre el aprendizaje y el conocimiento. Como se resaltó en este documento, las recientes demandas indígenas cuestionan la ontología del conocimiento escolar y, al parecer, esto también ocurre en distintos ámbitos de la vida cotidiana de las comunidades indígenas (cf. Stobart y Howard 2002).

Además, las condiciones disímiles que marcan la existencia de los idiomas involucrados en los programas de educación bilingüe indígena en Latinoamérica nos confron- 
tan con la situación de emprender varias tareas simultáneamente, pero también al mismo tiempo con diferentes objetivos en mente. Los idiomas indígenas no solo requieren usarse con propósitos pedagógicos específicos, sino también para contribuir a su propio bienestar y desarrollo; al hacerlo, se cuestiona inmediatamente la relación entre la oralidad ancestral y el alfabetismo moderno, así como entre la expresión oral y escrita en la escuela, y se nos pide adoptar posiciones claras respecto a la naturaleza de la escuela, como la agencia por excelencia para extender la escritura alfabética en toda América Latina.

Al mismo tiempo, los educadores bilingües necesitan asumir responsabilidades que trasciendan la enseñanza del lenguaje para involucrarse en actividades que contribuyan a la modernización, cultivo y uso extendido de los idiomas indígenas. Los educadores bilingües no solamente enseñan el idioma indígena sino que también lo transforman, , al usarlo en un contexto diferente a aquél del cual emergió y en donde se lo usa tradicionalmente. Esto se hace más evidente cuando uno considera que muchas de estas comunidades y lenguas conocieron solo recientemente la escritura alfabética (cf. Francis y Reyhner 2002; López 2001). En otras palabras, los educadores bilingües deben involucrarse en una serie de actividades que van mucho más allá del aula y de la enseñanza; necesitan actuar al mismo tiempo como maestros, como planificadores y también como activistas y promotores de los idiomas indígenas.

De ser correcto este análisis, muchas de las teorías y modelos que nos llevaron a los planificadores del lenguaje y a los especialistas en educación lingüística a las comunidades indígenas también deberán examinarse seriamente en estrecho diálogo e interacción con sus líderes y con los hablantes de las lenguas concernidas en general. Una de las cuestiones bajo consideración es la dicotomía transición-mantenimiento. ¿Qué podría significar esta oposición y 
qué es lo que implica en comunidades donde los niños solo completan 4 ó 5 años de escolaridad? ¿Deberían restringirse las consideraciones subyacentes a la educación bilingüe a la duración del programa o se necesita entender la pregunta de manera más amplia y más con relación a la calidad de la educación idiomática y en términos de las competencias lingüísticas y sociolingüísticas y la conciencia lingüística crítica (cf. Fairclough 1992) que los alumnos indígenas deberían desarrollar en el limitado período de tiempo escolar disponible en la mayoría de las escuelas rurales de la región? Bajo dichas limitaciones, ¿dónde debería colocarse el énfasis respecto a la enseñanza del segundo y/o tercer idioma? ¿No tendría quizá que prestarse más atención a fortalecer las competencias lingüísticas, sociolingüísticas y culturales de los estudiantes en su lengua materna? En casos como los que aquí se discuten, la respuesta podría muy bien ser la última pues se dispone ahora de amplia evidencia en la región respecto a la capacidad que desarrollan los alumnos indígenas de transferir a su segundo idioma algunas de las competencias claves desarrolladas en su primer idioma (cf. Francis y Reyhner 2002, López 1998, Dutcher 1995; Francis y Hamel 1992).

Evidencia de ello es que pese a que, en general, se dedica menos tiempo semanal a la enseñanza del segundo idioma, los niños indígenas en programas de educación bilingüe salen comparativamente mejor en las pruebas de castellano que sus pares escolarizados exclusivamente en esta lengua. A veces incluso la producción escrita en castellano puede sobrepasar el nivel de la capacidad oral en este idioma (cf. López y Jung 2003).

He aprendido a leer y escribir en quechua desde primero de primaria hasta sexto curso. Más que todo hemos aplicado puro quechua. La EIB me gustó mucho. Pero en sí, yo no he podido dominar la lengua castellana. Cuando me vine de octavo de primaria a cursar primero de medio, aquí 
en Mizque, tuve muchos problemas. [Pero] ahora estoy bien, he aprendido el castellano y me dicen que soy un autodidacta. Estoy alegre y me siento bien, porque a la vez sé escribir y leer también en quechua. En mis estudios, ahora, estoy muy bien también. Estoy entre los mejores, porque desde el primero medio, siempre he tenido diplomas. (Ramber Molina, estudiante quechua, Entrevista, marzo.2004).

En un contexto donde los teóricos de la planificación del lenguaje y la EIB y sus practicantes y promotores adoptaron la noción sugerente y políticamente provocadora de diglosia conflictiva tomada de la sociolingüística vasca y catalana (cf. Ninyoles 1972, Hamel y Sierra 1983) podría ser también necesario evaluar las ganancias y pérdidas de la aplicación de estas ideas seminales en las condiciones sociolingüísticas específicas de la América indígena. Cuando la EIB surgió de lo que originalmente eran proyectos educativos para estimular la rápida diseminación del castellano en los territorios indígenas, en primera instancia, los ideales de mantener y desarrollar el idioma indígena y luego los de enriquecer a la sociedad extendiendo el bilingüismo o multilingüismo conllevaron análisis sociolingüísticos relativos a la necesidad política de relocalizar los idiomas y las culturas indígenas tanto en los territorios indígenas como entre la sociedad hegemónica. En dicho contexto que hasta cierto punto es similar al experimentado en España a principios de los años 70 cuando se reinstituyó el régimen democrático, los planificadores del lenguaje y de la EIB latinoamericana adoptaron la noción de la normalización idiomática (Ninyoles 1972). Con este concepto, vascos y catalanes cuestionaron la asimetría que había favorecido históricamente al castellano y apuntaron a restablecer el equilibrio perdido entre el castellano y los idiomas ancestrales de las regiones autónomas vascas y catalanas. 
En forma similar, en Latinoamérica la introducción de la EIB -particularmente desde el marco del mantenimiento y desarrollo de los idiomas indígenas- proporcionó el contexto apropiado para cuestionar los roles y funciones que tendrían tanto el castellano como los idiomas indígenas en una sociedad culturalmente más democrática. Todos los esfuerzos se dirigieron a modernizar los idiomas indígenas, desarrollando sistemas de escritura y elaborando sus léxicos para contribuir a su estandarización y uso escolar y académico. De allí que la EIB y el aula fueron considerados un medio para reinscribir social y políticamente los idiomas indígenas en la sociedad moderna, para extender su uso a nuevos dominios sociales y para cumplir con nuevas funciones de comunicación. Se esperaba que los dos idiomas -el indígena y el castellano- pudieran usarse alternadamente a través de la escuela primaria. Las demandas colocadas sobre los idiomas indígenas fueron vastas y se hizo un esfuerzo enorme por intelectualizarlos (cf. Garvin y Matthiot 1958), irónicamente en detrimento de la enseñanza del castellano como segundo idioma, una tarea que demandaban las comunidades indígenas en donde se implementaba la EIB.

Después de dos décadas, puede haber llegado el momento de reconsiderar la noción de diglosia conflictiva, para identificar con las mismas comunidades indígenas el tipo de complementación que ellos desean establecer entre los dos o tres idiomas que hablan o que desearían que sus niños hablaran, leyesen o escribieran. En primer lugar, se podría cuestionar la validez del enfoque seguido por teóricos y practicantes de la EIB para estandarizar el idioma indígena, pues con frecuencia reclaman que el estandarizar un idioma indígena implica al mismo tiempo ir en desmedro las variedades específicas que hablan y que generalmente consideran estrechamente ligadas a su identidad étnica local y específica. No obstante, se debe reconocer al mismo 
tiempo la existencia de posiciones políticas claramente definidas que ven en los sistemas unificados de escritura y en la estandarización un mecanismo para fortalecer la identidad indígena diferenciada y la construcción paulatina de un sentido nacional indígena.

Empero, al mismo tiempo, la evolución de la ideología de la EIB nos lleva a distanciarnos de los nítidos márgenes entre tradición y modernidad que, respecto a la problemática del lenguaje, ciertamente evitarán que restrinjamos el uso de idiomas indígenas a los dominios tradicionales, a la vez que empleamos los idiomas más hablados en los así llamados contextos modernos. Esta es una frontera que los líderes indígenas usualmente rechazan, pues para sobrevivir han tenido que reubicarse en "las capas complejas y fluidas de la interacción" (Howard, Barbira-Freedman y Stobart 2002:2) que hoy en día caracterizan la comunicación en las comunidades indígenas. En una situación tal, tradición y modernidad se complementan y el conocimiento contemporáneo y otros elementos culturales podrían también ser incorporados a la matriz cultural indígena.

Hace unos cuantos años, un reconocido intelectual indígena le dijo a este autor: "Yo hago caso omiso de la división que ustedes establecen entre tradición y modernidad. Nosotros también hemos tenido nuestra propia modernidad, el problema es que la nuestra ha sido diferente a la de ustedes en tanto parte de una historia diferente" (Víctor Hugo Cárdenas, comunicación personal) ${ }^{19}$. Son estas otras historias las que tenemos que ayudar a reconstruir y comprender, si queremos reinventar la EIB para que sirva de mejor forma a estas relocalizadas sociedades multilingües.

Esto es particularmente imperativo hoy que los líderes y las organizaciones indígenas presentan a la sociedad

\footnotetext{
${ }^{19}$ Pedagogo aimara y especialista en educación intercultural bilingüe, Cárdenas fue elegido vicepresidente de Bolivia para el período 1993-1997.
} 
hegemónica sus propuestas haciendo uso de un castellano oral y escrito, apropiado y bien elaborado, y, en casos como el de Guatemala, en libros publicados por editoriales mayas. Empero, cuando necesitan hacerlo ante sus pares analizan y presentan las problemáticas en cuestión en un aimara, maya, quechua o guaraní de igual calidad comparable a la de "su" castellano, o a veces también se mueven entre un idioma y otro, cuando saben que sus interlocutores son bilingües.

Cuando a principios del siglo XIX los líderes indígenas se adscribían a la legislación actual, que reconocía la igualdad ante la ley y el derecho de todos los ciudadanos a la educación, construyeron sus propias escuelas e inicialmente pagaron a sus maestros; una vez que las escuelas estuvieron establecidas y funcionando exigieron que el Estado asignase plazas docentes con cargo al presupuesto nacional. Hoy en día -también recurriendo a la legislación nacional, la palabra escrita y el idioma hegemónicodemandan su derecho a la diversidad y a ser ellos mismos en el marco de un Estado plurinacional, donde las escuelas son vistas como el instrumento público responsable de promover la interculturalidad, el bilingüismo y el multilingüismo.

Al hacerlo, formulan propuestas que van mucho más allá de sus intereses políticos y étnicos específicos, en un intento por llevar sus idiomas y culturas al cauce de lo hegemónico, en un renovado marco de una democracia intercultural. Los líderes indígenas reiterativamente le recuerdan a la sociedad en general que el multiculturalismo y el multilingüismo no son características del pasado sino más bien rasgos del futuro que están relacionados con las competencias que todos los latinoamericanos necesitan desarrollar para ser ciudadanos del mundo. Como ellos reafirman muchas veces, los indígenas, desde siempre, han tenido que adoptar una actitud intercultural y multilingüe para poder sobrevivir. Puede haber llegado el momento en el 
cual los individuos que no somos indígenas debamos aprender de ellos para convertirnos en multilingües y así enfrentar de mejor modo el nuevo mundo que se presenta ante nosotros en América Latina. Para adaptarnos a estas nuevas condiciones y desafíos políticos y sociales no hay otra alternativa que imaginar una nueva y remozada EIB para un mundo que sea verdaderamente multilingüe e intercultural. 
